Annuaire suisse de politique de développement

Société de l'information et coopération internationale

\title{
La Suisse et le sommet mondial sur la société de l'information : défis et déficits
}

Fabrice Boulé

\section{(2) OpenEdition}

1 Journals

Édition électronique

URL : http://journals.openedition.org/aspd/575

DOI : $10.4000 /$ aspd. 575

ISSN : 1663-9669

Éditeur

Institut de hautes études internationales et du développement

Édition imprimée

Date de publication : 1 novembre 2003

Pagination : 151-156

ISSN : 1660-5934

\section{Référence électronique}

Fabrice Boulé, "La Suisse et le sommet mondial sur la société de l'information : défis et déficits »,

Annuaire suisse de politique de développement [En ligne], 22-2 | 2003, mis en ligne le 22 mars 2010, consulté le 08 septembre 2020. URL : http://journals.openedition.org/aspd/575 ; DOI : https://doi.org/ 10.4000/aspd.575 


\title{
La Suisse et le sommet mondial sur la société de l'information: défis et déficits
}

\author{
Fabrice Boulé*
}

e Sommet mondial sur la société de l'information (SMSI) met la Suisse au pied du mur. Les promoteurs de la candidature de Genève ont vu loin: une opportunité politique pour la ville onusienne et le renforcement du rôle international de la Suisse sur des thèmes porteurs durant les prochaines décennies. C'est la première fois que la communauté internationale traite de la communication et de l'information à un tel niveau. Pour accentuer la différence avec d'autres sommets, des règles nouvelles ont été annoncées: la société et le secteur privé doivent pouvoir se rapprocher sensiblement des décideurs politiques. C'est une tendance officialisée et encouragée par l'ONU depuis plusieurs années. Mais Genève 2003 constitue un test crucial pour cette collaboration: le sommet veut en effet exprimer une vision commune de la société de l'information de demain, partagée par les Etats, la société civile et les acteurs économiques.

Dans la longue valse diplomatique qui caractérise la préparation d'un tel sommet, être le pays hôte est à la fois une chance et un risque: on a une marge de manœuvre pour montrer la voie et faire des propositions, mais on sera aussi tenu pour largement responsable en cas d'échec. Après son adhésion à l'ONU en septembre 2002, la Suisse doit faire preuve de son savoir-faire onusien en préparant un sommet alléchant. En cas de réussite, elle sera un nouvel acteur d'autant plus écouté sur les autres sujets qui lui tiennent à cœur: promotion du droit humanitaire, de la sécurité humaine (lutte contre les mines antipersonnel, le commerce des armes légères, le recrutement des enfants soldats), défense des droits de l'homme (la Suisse envisage de se porter candidate en 2007 à la commission ad hoc), protection de l'environnement (défense des principes de précaution et du pollueur payeur).

A quoi se mesurera le succès? A une logistique sans faille? Les préparateurs suisses y tiennent beaucoup; c'est une image de marque. Au nombre et à l'envergure des chefs d'Etat et de gouvernement qui feront le déplacement? Sans nul doute, puisque cette affluence déterminera largement la résonance médiatique de l'événement. Mais les visiteurs de marque n'afflueront que si le programme est attrayant, porteur politiquement, bon pour l'image... sans mettre personne trop profondément dans l'embarras. Les infrastructures de communication et la nature de l'information devront, théoriquement, contribuer à atteindre les objectifs formulés par l'ONU dans sa Déclaration du millénaire en 2000: réduction de la pauvreté, accès aux soins, à l'éducation, bonne gouvernance,

* Journaliste à InfoSud et secrétaire général de Syfia International, Suisse. 
valorisation du rôle des femmes, etc. Il s'agit donc de trouver le plus grand dénominateur commun entre les gouvernements, le secteur privé et la société civile, en jonglant avec des sujets hautement sensibles tels que la liberté d'expression, l'accès à l'information comme un bien public, et encore les règles de propriété intellectuelle, etc. Depuis le début de l'année, Marc Furrer, directeur de l'Office fédéral de la communication (OFCOM), maître d'œuvre du sommet côté suisse, est monté au créneau pour promouvoir une réunion ouverte et innovatrice: société civile et secteur privé doivent participer activement et codécider pour que le sommet soit un succès.

A Rio, en 1992, l'ONU avait mobilisé longtemps à l'avance un «Monsieur Sommet de la Terre», Maurice Strong, qui s'était entouré de dizaines de collaborateurs. Ils ont rendu opérationnel le concept du «développement durable», formulé initialement en 1987 dans le rapport Brundtland. Rio a été un succès car un esprit de Rio a été façonné avant le sommet. Pour Genève, rien de tel. Aucun leadership n'a été recherché, aucune personnalité n'a été profilée pour faire émerger un esprit de Genève et faire du SMSI un «Rio de l'information». La Suisse n'a pas produit sa propre vision de la société de l'information de demain et n'a donc pas pu mobiliser ses troupes et celles d'éventuels alliés de poids derrière cet étendard. Il ne s'agissait pas d'être la grenouille à peine débarquée à l'ONU et qui veut ressembler à un bœuf, mais de rassembler des visionnaires, d'en faire bouillir les idées et de servir aux convives un menu stimulant.

Remontons à mai 2002, lors de la conférence africaine de préparation du sommet tenue à Bamako, la capitale malienne. Avec l'Union européenne et la Francophonie, la Suisse a largement financé ce premier rendez-vous. Walter Fust, directeur de la coopération suisse (DDC), y exprimait sa vision: un grand sommet de communication sociale plutôt qu'une conférence technique. Et surtout pas un simple concours de beauté entre Etats. Mais les craintes de trop se mettre en avant, de trop s'exposer apparaissaient déjà. «Attention, le SMSI n'est pas un sommet suisse, mais un sommet onusien, avertissait Fust. Beaucoup de pays africains font la confusion. C'est l'ONU qui doit le promouvoir auprès des Etats et force est de constater qu'elle n'a pas encore vraiment commencé.» Dix-huit mois plus tard, l'ONU n'a toujours pas vraiment entamé cette tâche. Et la Suisse, par prudence ou indécision, aura laissé passer l'occasion de prendre les choses en main.

On peut certes trouver différentes raisons à cette absence d'axe fort pour le SMSI. Elles ont à voir autant avec le contexte international de 2003 qu' avec les méandres onusiens, fédéraux et cantonaux qui ont compliqué les préparatifs :

$\checkmark$ Le «made in Switzerland», en termes de dispersion des responsabilités entre différents offices fédéraux, d'absence de direction politique claire, de désintérêt persistant ou d'ignorance de l'importance de l'enjeu, a pesé lourd. Au printemps 2003, on a tenté de remédier à ces handicaps, mais beaucoup de temps avait déjà été perdu.

$\square$ Le tout antiterrorisme post-11 septembre 2001, la guerre en Afghanistan, la guerre en Irak n'ont pas aidé à réunir les bonnes volontés pour réfléchir au partage de la connaissance au XXI siècle. 


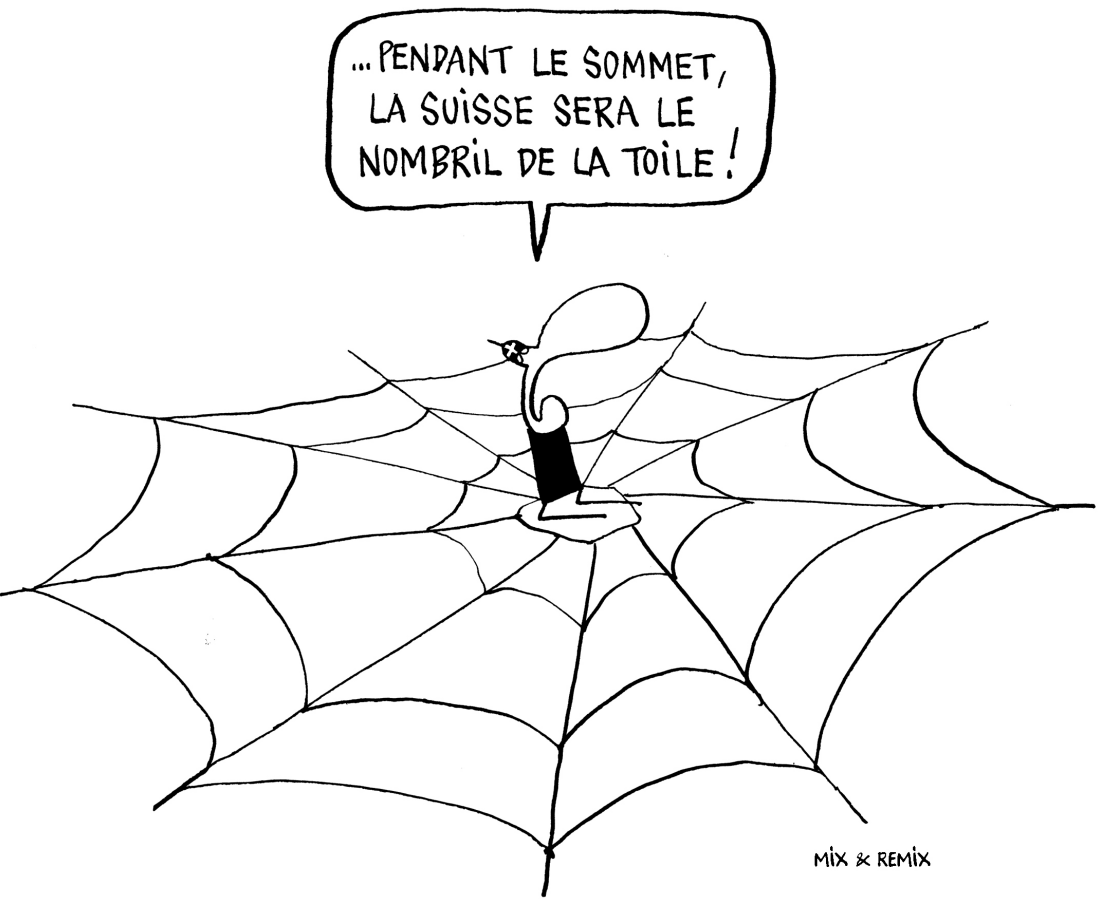

$\square \quad$ Les tergiversations de Yoshio Utsumi, le secrétaire général de l'Union internationale des télécommunications (UIT), autour des candidatures de Genève et de Tunis ont retenu tout le monde dans les starting-blocks pendant plus de dix mois. Notons que la Tunisie pensait depuis quelques années à un projet de sommet sur l'information. C'est elle qui a déposé en 1998 une résolution lors du Congrès de l'UIT à Minneapolis, demandant qu'on étudie la faisabilité d'un tel sommet. L'adoption de cette résolution a ouvert la voie vers le SMSI. Dans un premier temps toutefois, on ne prévoyait qu'un sommet de l'UIT, en 2002, avec les gouvernements et l'industrie, mais sans la société civile. Mais en 2001, une résolution de l'Assemblée générale de l'ONU a souhaité la pleine participation de la société civile.

$\square \quad$ L'attribution de la deuxième phase à Tunis pose évidemment un problème. Ce pays brille par l'étouffement complet de la liberté d'expression. Au moment où se tenait le $2^{\mathrm{e}}$ Prepcom (comité préparatoire), plusieurs internautes étaient arrêtés par les autorités tunisiennes pour avoir accédé à des sites politiquement incorrects. Les opposants demandent au régime, sans trop y croire, de donner des signaux d'ouverture pour mériter les honneurs de 2005. A défaut, ce suivi du SMSI à Tunis devrait fournir aux militants tunisiens des droits de l'homme une tribune de choix pour se faire entendre. 
$\square$ Ce partenariat forcé entre la Suisse et la Tunisie a parfois produit des perspectives burlesques. Début 2003, l'ancien ministre malien Adama Samassekou, qui préside le processus préparatoire, lance une idée pour battre le rappel avant décembre: pourquoi ne pas organiser à Genève une rencontre des présidents des pays hôtes du sommet et des pays qui ont accueilli les conférences régionales de préparation? Quelques mauvais esprits ont immédiatement imaginé la poignée de mains, bien au centre de la photo, entre Pascal Couchepin et le président tunisien Ben Ali, pour vendre la société de l'information de demain. Ce projet n'est plus remonté à la surface. Officiellement, bien sûr, Berne et Tunis sont à l'unisson.

$\checkmark \quad$ L'UIT, chargée par l'ONU de coordonner la préparation du SMSI, est une agence technique. Elle n'a pas l'expérience de négociations multilatérales de nature politique. Basée à Genève, elle y organise traditionnellement le salon Télécom. De plus, l'UIT travaille étroitement avec les entreprises privées, mais n'a jamais eu affaire auparavant à la société civile. Beaucoup d'acteurs suisses du SMSI n'ont cessé d'accuser l'UIT d'inactivité, voire de manœuvres d'obstruction au nom d'un droit de contrôle.

$\checkmark$ Beaucoup d'acteurs suisses se sont plaints également du très faible engagement financier de l'UIT. De son côté, Berne a débloqué quelque 20 millions de francs. Environ 15 millions seront consacrés à la sécurité, à la logistique et à l'organisation du sommet (les postes de deux membres du Secrétariat exécutif, celui du délégué du Conseil fédéral, les frais d'organisation des prepcoms). Entre 5 et 6 millions de francs devraient également être déboursés dans des manifestations parallèles, organisées par la DDC, le Secrétariat à l'économie (seco), les Offices fédéraux de la culture, de la statistique, etc. Le canton de Genève a, quant à lui, avancé un chiffre total de 2 à 3 millions de francs: environ 1 million pour la logistique, la sécurité et le protocole, et entre 1 et 2 millions pour le volet société civile: les postes de deux personnes à la division Société civile du Bureau du sommet pour quelque deux années et des frais logistiques pour faciliter la présence et l'expression d'organisations non gouvernementales.

$\square \quad$ La crise que traverse le secteur économique des télécoms et de l'informatique ne facilite pas la mobilisation des entreprises de ce secteur. Leur attentisme affaiblit le caractère tripartite du SMSI (gouvernements et organisations internationales - société civile - secteur privé).

$\checkmark$ Pourquoi l'Office fédéral de la communication (OFCOM) a-t-il été désigné comme maître d'œuvre plutôt que le Département des affaires étrangères (DFAE)? A Berne, il ne semble pas y avoir eu de débat approfondi sur la question. L'OFCOM étant membre de l'UIT, il y avait une certaine logique à ce qu'il prenne les commandes. C'est lui aussi qui coordonne le Comité interdépartemental pour la société de l'information (CISI), chargé du pilotage des activités fédérales en matière de technologies de l'information et de la communication (TIC). Il fait chaque année un état des lieux à l'attention du Conseil fédéral. A l'époque de l'attribution des rôles, au début de l'été 2001, le DFAE de Joseph Deiss était plongé dans la campagne pour l'adhésion à l'ONU. Il n'aurait donc eu ni l'énergie ni l'argent nécessaires pour prendre en main la préparation du SMSI. Il ne s'est en tout cas pas battu pour l'obtenir, privant ainsi l'organisation du sommet d'une mobilisation 
précoce de la diplomatie suisse à travers le monde. La DDC, très active dans la promotion des TIC pour le développement, a pu évidemment être tentée, mais elle ne l'a pas fait savoir. Le SMSI n'est pas à proprement parler un sommet sur le développement. De plus la DDC n'avait aucune envie de courir le risque de devoir payer la facture pour une réunion de techniciens des télécoms. Finalement, elle a jeté tout son dévolu sur l'organisation d'un événement parallèle au SMSI. Elle entend attirer à Genève les meilleurs exemples d'utilisation des TIC pour le développement social et économique (ICT4D).

- Une star africaine s'est décommandée au dernier moment. Depuis longtemps, deux acteurs genevois du SMSI - Alain Clerc, qui dirige la division Société civile au Secrétariat exécutif, et l'ex-conseiller d'Etat Guy-Olivier Segond, ambassadeur spécial de l'UIT pour le SMSI - avaient imaginé un rôle privilégié pour leur ami Alpha Oumar Konaré, président du Mali jusqu'en 2002. Il a préféré renoncer à présider le processus de préparation du sommet, plus tenté par la perspective de diriger la nouvelle Union africaine (ex-OUA). C'est un ancien ministre malien de l'Education, Adama Samassekou, qui a finalement été désigné. L'absence du président Konaré, l'homme qui voulait connecter toutes les communes de son pays à Internet, a donc certainement pesé en termes de visibilité. Moins médiatique, son remplaçant de dernière heure accomplit cependant un travail actif et efficace. Au Mali, il préside l'Académie africaine des langues, qui fait l'inventaire des multiples langues parlées en Afrique de l'Ouest. Une expérience intéressante pour un sommet qui entend aussi traiter la diversité linguistique et culturelle sur le Net.

L'axe Genève-Bamako, ou Genève-Afrique, pèse lourd dans la genèse du SMSI. Nelson Mandela a lancé le thème à l'ouverture de Telecom 1995 : loin de combler le fossé Nord-Sud, les nouvelles technologies éloignaient encore plus vite les «infopauvres» des «inforiches». L'année suivante, Genève organise une conférence sur l'Afrique et les nouvelles technologies. Invité de marque, Alpha Oumar Konaré séduit les intervenants de la société civile. Un rêve est né: et si les gouvernements débattaient sur pied d'égalité avec les syndicats, les femmes, les jeunes, les chercheurs, les chefs d'entreprise? La Fondation du devenir, dirigée notamment par Alain Clerc, organise la conférence «Bamako 2000 : Internet, les passerelles du développement», avec l'appui financier du Canton de Genève et de la DDC. Les représentants de 40 Etats, les délégués de la société civile et les représentants du secteur privé se mélangent joyeusement. L'UIT est là aussi. Dans la foulée, elle demande aux Genevois de prendre en main le projet de sommet décidé en 1998, mais resté en panne. La société civile entre de plain-pied dans les plans du futur SMSI. Un secrétariat tripartite du sommet est mis sur pied. La division Société civile est financée par le Canton de Genève.

Les Genevois le martèlent: le SMSI sera le premier sommet d'une nouvelle génération, reposant sur les forces vives de la société réelle - l'engagement citoyen et le dynamisme du secteur privé. Les gouvernements ne peuvent plus décider tout seuls; c'est particulièrement vrai quand on parle information et communication. Mais en juillet 2002, la société civile reçoit une douche froide: au $1^{\text {er }}$ Prepcom, les gouvernements ne lui ouvrent pas la porte; les ONG piaffent 
d'impatience dans l'antichambre. Auraient-elles été trompées sur la marchandise? Les promesses étaient sans doute exagérées et le slogan "sommet sans précédent» peut-être trop vendeur. Il est vrai que l'association des Etats, des ONG et des entreprises lors des sommets onusiens n'a pas été inventée à Genève. Elle est en marche depuis des années. L'évolution des mentalités des Etats est lente, mais indiscutable. De sommet en sommet, les règles de procédure évoluent.

Par la suite, les choses se sont un peu arrangées. Lors de la deuxième réunion préparatoire, en février 2003, les membres de la société civile ont pu assister aux groupes de travail. Ils ont même pu proposer des modifications à l'esquisse de déclaration de principe et de plan d'action. Les gouvernements restent cependant libres d'accepter ou non ces propositions, qui figurent dans un document séparé, mais néanmoins officiel. Au même moment, un Bureau de la société civile est né, structure permanente en vue du sommet et même appelée à demeurer par la suite à Genève. A condition bien sûr qu'elle soit financée. «Un bureau de la société civile, organisée et reconnue comme interlocuteur légitime, pourrait mettre fin à l'opposition binaire et stérile entre les salons des diplomates et les "manifs" des rues », observe alors Alain Clerc. En parallèle, la société civile suisse avance ses pions. En février, une plate-forme d'organisations non gouvernementales, d'organisations professionnelles et des médias a appelé Berne à réagir. Frustrée, la société civile estime que la dynamique innovatrice du sommet est en panne. Brandissant la menace d'un contre-sommet, elle demande aussi la mise en place d'un groupe de réflexion de haut niveau regroupant l'administration fédérale, des parlementaires, la société civile, les médias et le secteur privé. Le principe d'une collaboration accrue a été accepté par Berne qui a cependant mis un frein aux velléités des ONG: oui à une consultation plus intensive, non à une reconnaissance institutionnelle paritaire de la société civile, dont les membres ne sont pas élus démocratiquement. 